\title{
Morphological Studies on Proliferation and Desquamation of the Alveolar Lining Epithelium in Carrageenan-Induced Experimental Pneumonia
}

\author{
Takehiro Mitsuhashi, Masayoshi Shimazaki, Seiki Sugino, \\ Tadashi Takeda and Hiromi Inariba \\ Department of Pathology, Osaka City University Medical \\ School, Osaka 545
}

\begin{abstract}
Mitsuhashi, T., Shimazaki, M., Sugino, S., Takeda, T. and Inariba, H. Morphological Studies on Proliferation and Desquamation of the Alveolar Lining Epithelium in Carrageenan-Induced Experimental Pneumonia. Tohoku J. exp. Med., 1983, 140 (3), 319-330 — An experimental model of carrageenan-induced pneumonia in rabbits was used to study the proliferation and desquamation of alveolar lining epithelium, light and electron microscopically. Observation at $5 \mathrm{hr}$ after intrapulmonary injection of $0.5 \%$ lambda carrageenan solution revealed epithelial cell (predominantly type 1 pneumocyte) injury, exposing the basement membrane of the alveolar wall, and that at $40 \mathrm{hr}$ demonstrated an appearance of many large pneumocytes among the alveolar lining epithelium as a reparative reaction. This proliferative response of immature type 2 pneumocytes reached to the maximum in 60 and $72 \mathrm{hr}$ followed by an intense desquamative response of the excessive alveolar epithelium in 5 and 6 days. The sloughed off epithelial cells were demonstrated as cell sheets or as clusters of epithelial cells. Inflammatory infiltrates in alveolar and interstitial spaces consisted of neutrophils and monocytes (or macrophages) at early stages, and mononuclear cells or macrophages at latter stages. Observation in 2nd and 3rd week showed accumulation of macrophages with many osmiophilic lamellar bodies in the alveolar spaces. The alveolar wall lining epithelium was still cuboidal or elliptical in shape in some alveoli and membranous in the others. Thickened alveolar septa at this stage were also noted. Thus this animal model appears to be a valuable tool for further understanding of the events leading to proliferation and desquamation of the alveolar lining epithelium. carrageenan; type 1 pneumocyte injury; type 2 pneumocyte proliferation; type 2 pneumocyte desquamation
\end{abstract}

Carrageenans comprise a variety of sulphated polygalactans extracted from marine algae, which have several well-known biological properties. Induction of acute and chronic inflammatory responses is one of those properties. Extensive studies have been performed on the inflammatory response provoked by carrageenans and the analysis of the mechanism underlying this response. Recently, carrageenans have also been used to induce inflammation in the lungs of experimental animals. The morphological changes in carrageenan lungs have been described as the lesion resembling human lobar pneumonia (Trenchard et al. 1972;

Received for publication, November 22, 1982. 
Velo and Spector 1973) and as a catarrhal suppurative broncho-pneumonia (an aspiration pneumonia) (Szereda-Przestaszewska and Sieiński 1979; Wachtlová et al. 1975). Despite these reports concerning inflammatory responses of the lungs, there has been no detailed description of the effects of carrageenans on the alveolar lining epithelium. In the course of histological studies on carrageenan lungs carried out in our laboratory, prominent proliferation of alveolar lining epithelial cells followed clearly by an intense epithelial cell desquamation like the histological findings in desquamative interstitial pneumonia were recognized. To our knowledge, there are few reports on experimental animal model which could induce such an intense proliferation and desquamation of alveolar lining epithelium, although some proliferation of the lining epithelium, usually termed "alveolar epithelization" (Alley and Manktelow 1971), is a common feature of mammalian pneumonia.

The present report details the histopathologic and ultrastructural studies focused on the morphological changes of these epithelial cells in the course of the carrageenan-induced pneumonia.

\section{Materials and Methods}

Animals. Healthy male albino rabbits, 2 to $2.5 \mathrm{~kg}$ in body weight, were used for this study.

Anesthesia. For injection of the irritants, anesthesia was achieved by intravenous injection of $20 \mathrm{mg}$ Nembutal (pentobarbital sodium) per $\mathrm{kg}$ body weight.

Irritant. Autoclaved $0.5 \%$ lambda carrageenan (Sigma Chemical Co., Lot No. C 3889) in physiologic saline was prepared and refrigerated until used. Sterile physiologic saline was used as a control. Inflammation was induced by the injection of lambda carrageenan into the lung by a method modified from the techniques of Trenchard et al. (1972) and Bowers et al. (1980). Briefly, the trachea was exposed through a mid-line neck incision. After making a tracheal incision, a firm flexible catheter (outer diameter $4 \mathrm{~mm}$, about $27 \mathrm{~cm}$ in length, with one end rounded and the other connected to a stainless steel gastric tube for rat and $10 \mathrm{ml}$ syringe) was inserted into the trachea and gently lowered into the lung until it wedged in a lower lobe. It was then withdrawn slightly, $8 \mathrm{ml}$ of carrageenan solution was injected. The catheter was thereupon gently withdrawn. The overlying muscles were allowed to rejoin across the trachea, the skin sutured and animals allowed to recover. Control animals were treated in exactly the same way except that only saline was injected into the lungs. One to three animals at specified time intervals $(5,40,60 \mathrm{and} 72 \mathrm{hr}$, 5 and 6 days, then 2 and 3 weeks) after administration of $0.5 \%$ lambda carrageenan solution or saline were sacrificed by intravenous injection of $2.5 \%$ glutaraldehyde fixative in $0.1 \mathrm{M}$ phosphate buffer, $\mathrm{pH} 7.4$ under light anesthesia. The trachea of each animal was exposed through mid-line neck incision and was pinched shut. The lungs, thymus and heart were dissected out together. The pathological lobes in the experimental (which can be distinguished at the gross level) and all the control lobes were split longitudinally to the long axis, to aid in penetration of fixative. Specimens for light microscopy were fixed in $20 \%$ neutral buffered fromalin solution and embedded in paraffin. Sections were cut at $4 \mu \mathrm{m}$ and stained with hematoxylin and eosin. Selective sections were stained by the periodic acid-Schiff method and by Giemsa stain. For electron microscopy, $1 \mathrm{~mm}$ cubes of the lesions obtained from pathological lobes in experimental and control lobes were fixed in $2.5 \%$ glutaraldehyde for about $2 \mathrm{hr}$ at $0^{\circ} \mathrm{C}$ and post-fixed in $1 \%$ osmium tetroxide in phosphate buffer $\left(\mathrm{pH} \mathrm{7.4)}\right.$ at $0^{\circ} \mathrm{C}$ for about $2 \mathrm{hr}$. The tissue blocks were then placed in a graded series of alcohol solutions and embedded in Poly/Bed 812 resin (Poly- 
sciences, Inc., Warrington, PA, USA). Thick sections were cut, then stained with toluidine blue and ultra-thin sections were made with glass knives and doubly stained with uranyl acetate and lead nitrate (Reynolds 1963). The approval sections were examined in a Hitachi H 300 transmission electron microscope.

\section{Results}

Observation at $5 \mathrm{hr}$. The inflammation was mainly localized in left lower lobes and the uninvolved lobes of the lungs were normal in appearance (as was the case for all subsequent hours, days and weeks). The histopathological changes found in animals exposed to carrageenan consisted of diffuse alveolar exudation of protein-rich fluid containing carrageenan which was stained a reddish purple with toluidine blue (Bowers et al. 1980) and detected with Giemsa stain (Velo and Spector 1972). It was accompanied by focal alveolar hemorrhage. Infiltrates of neutrophils in the interstitial and intra-alveolar areas were also seen. The bronchioles showed no abnormality.

Companion tissue examined by a transmission electron microscope revealed membranous pneumocyte injury, exposing the basement membrane, and a scattering of neutrophils in the intra-alveolar and interstitial compartments (Fig. 7).

Controls appeared almost normal for this time except for a few accumulations of alveolar macrophages, and for all the following sampling periods (Fig. 1).

Observation at $40 \mathrm{hr}$. Abundant edema fluid and infiltrates of alveolar macrophages and leukocytes were present in alveolar lumen and septum. Many large epithelial cells among the alveolar lining epithelium appeared (Fig. 2). The new epithelial cells were large-sometimes bizzare - , elliptical or cuboidal cells with amphophilic cytoplasm and large round to ovoid vesicular nuclei with one to two basophilic nucleoli, and were randomly distributed in the alveolar lining epithelium (Fig. 2). Piling up of the new epithelial cells into the alveolar lumen as appeared like lying bricks was noted. Mitoses of the new large cells were frequently seen. In only a few respiratory bronchioles epithelial cell hyperplasia was shown.

Ultrastructural studies of the new epithelial cells showed that they were larger than the mature type 2 pneumocyte. They possessed prominent nucleoli in euchromatic nuclei, and their cytoplasm contained numerous free ribosomes, but a few other organelles; osmiophilic inclusions are a few or absent (Fig. 8). The cell outline was irregular due to the presence of many short stubbly microvilli, although there were no fibrils in the villi as they were seen in type 3 pneumocytes (Merick and Reid 1970). The cell junction between these cells showed cellular invaginations and ultrastructural findings suggested that the new epithelial cells were immature type 2 pneumocytes derived from alveolar lining epithelium.

Observation at 60 and $72 \mathrm{hr}$. Sections from the lungs revealed a diffuse hypercellularity which, at a higher magnification, was associated with the prominent epithelial cell proliferation and the collection of inflammatory infiltrates such as neutrophils and macrophages (Fig. 3). Mitotic figures of the epithelial cells and sometimes macrophages were most frequently observed at this time. In the 


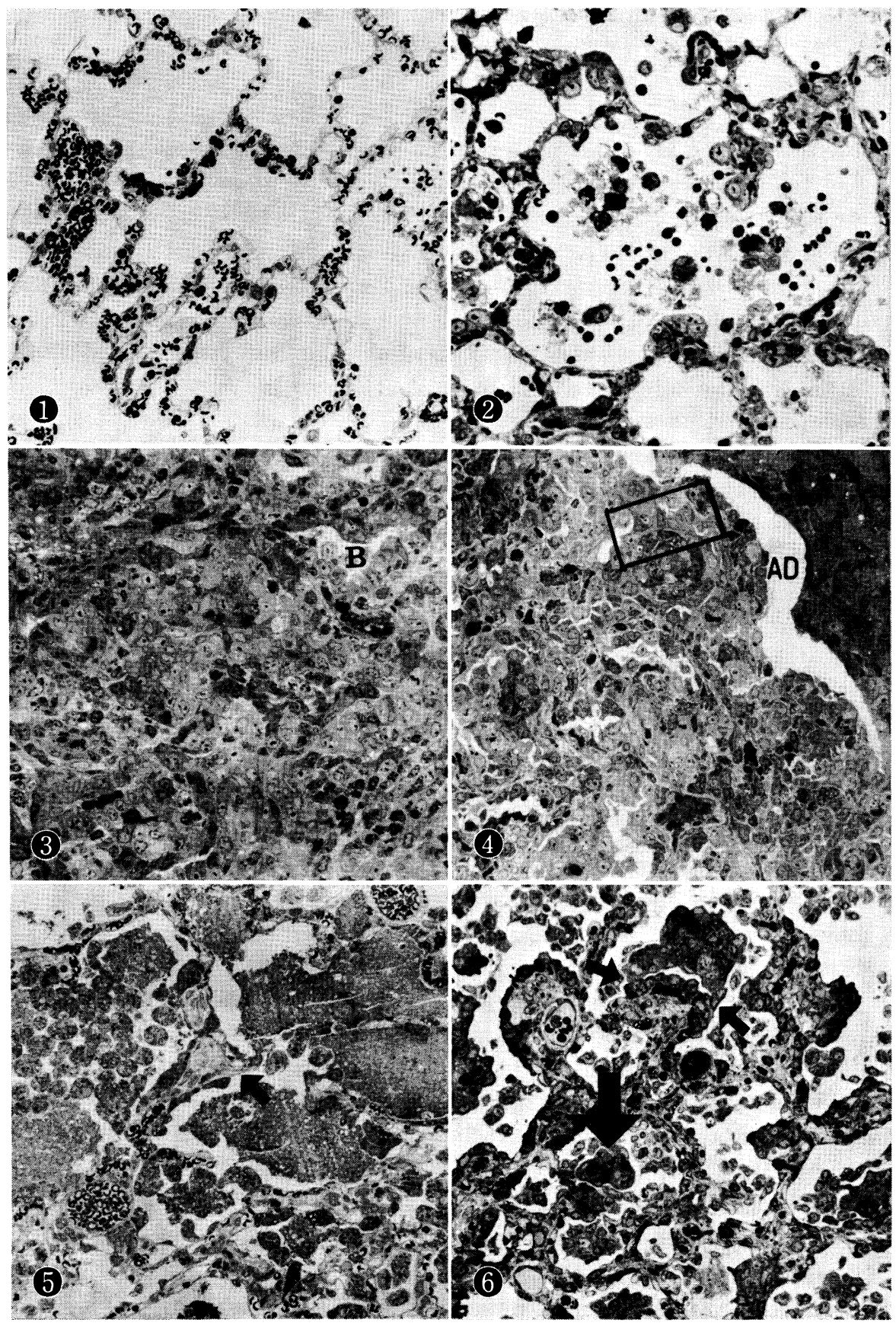


respiratory bronchioles some epithelial cell hyperplasia was also noted.

Ultrastructural studies confirmed the histological findings. Alveolar surface of the thickened wall with inflammatory infiltrates was jammed with a suprisingly large number of enlarged alveolar lining epithelial cells which had numerous microvilli on the luminal surface and cell junction complex between them (Fig. 9).

Observation on 5 and 6 days. Resin embedded thick sections stained by toluidine blue demonstrated that alveolar lumina were filled with infiltrated alveolar macrophages, cell debris and the newly proliferated and sloughed off epithelial cells. Those epithelial cells were revealed as a cluster of desquamated epithelial cells in some alveoli, as a epithelial cell sheet which often connected with alveolar wall lining epithelia only in a few parts in another (Fig. 5) and in the others as a cell mass in which the new epithelial cells proliferated into the alveolar lumen to pack up the cell debris and the many infiltrated alveolar macrophages (Fig. 4). The cytoplasm of alveolar macrophages in alveolar lumen was stained positive for the presence of carrageenan. A moderate increase in alveolar wall thickness with infiltration of mononuclear leukocytes and some increased connective tissue was shown. Some hyperplasia of the epithelial cells in respiratory bronchioles was seen.

Electron microscopic examination demonstrated in alveolar lumen an appearance of desquamated epithelial cells, which had numerous microvilli of their surface, interdigitations and tight junctions between them. Some of these desquamated pneumocytes were connected with alveolar wall lining epithelium only by a complex junction (Fig. 11). These findings resembled those of desquamated pneumocytes in human desquamative interstitial pneumonitis described and illustrated by Leroy (1969). The new pneumocytes which proliferated into the alveolar lumen to

Fig. 1. The lung tissue in a control rabbit $(5 \mathrm{hr}$ after intrapulmonary injection of physiologic saline). Resin embedded thick section, toluidine blue stain, $\times 200$.

Fig. 2. Epithelial regeneration in lung studied at $40 \mathrm{hr}$ after intrapulmonary injection of $0.5 \%$ lambda carrageenan solution. Many immature pneumocytes among the alveolar lining epithelium are easily recognized by their large size and cuboidal or elliptical shape. Resin embedded thick section, toluidine blue stain, $\times 240$.

Fig. 3. The most prominent findings at 60 and $72 \mathrm{hr}$ after $0.5 \%$ carrageenan treatment is widespread hyperplasia of immature type 2 pneumocytes. The figure shows diffuse hypercellularity. B, bronchiole. Resin embedded thick section, toluidine blue stain, $\times 240$.

Figs. 4 and 5. Epithelial desquamation in lung studied on 5 (Fig. 4) and 6 (Fig. 5) days after $0.5 \%$ carrageenan treatment. Fig. 4 shows narrow cracks between one layer of the alveolar wall lining epithelium and cell masses or cell clusters in the alveolar lumina. Alveolar duct (AD) is dilated and filled up with cell mass. Resin embedded thick section, toluidine blue stain, $\times 240$. Fig. 5 shows many alveolar spaces filled with alveolar macrophages and clusters of sloughed off epithelial cells (large arrow). Note a narrow cleft between alveolar wall lining epithelium and epithelial cell sheet (small arrow). Resin embedded thick section, toluidine blue stain, $\times 200$.

Fig. 6. A section from the lung tissue at 3 weeks after $0.5 \%$ carrageenan treatment showing amorphous granular materials and/or collection of alveolar macrophages in many alveoli. An increase in thickness of alveolar wall with a infiltrate of fibroblast-like mesenchymal cells is present (arrow). Resin embedded section, toluidine blue stain, $\times 200$. 


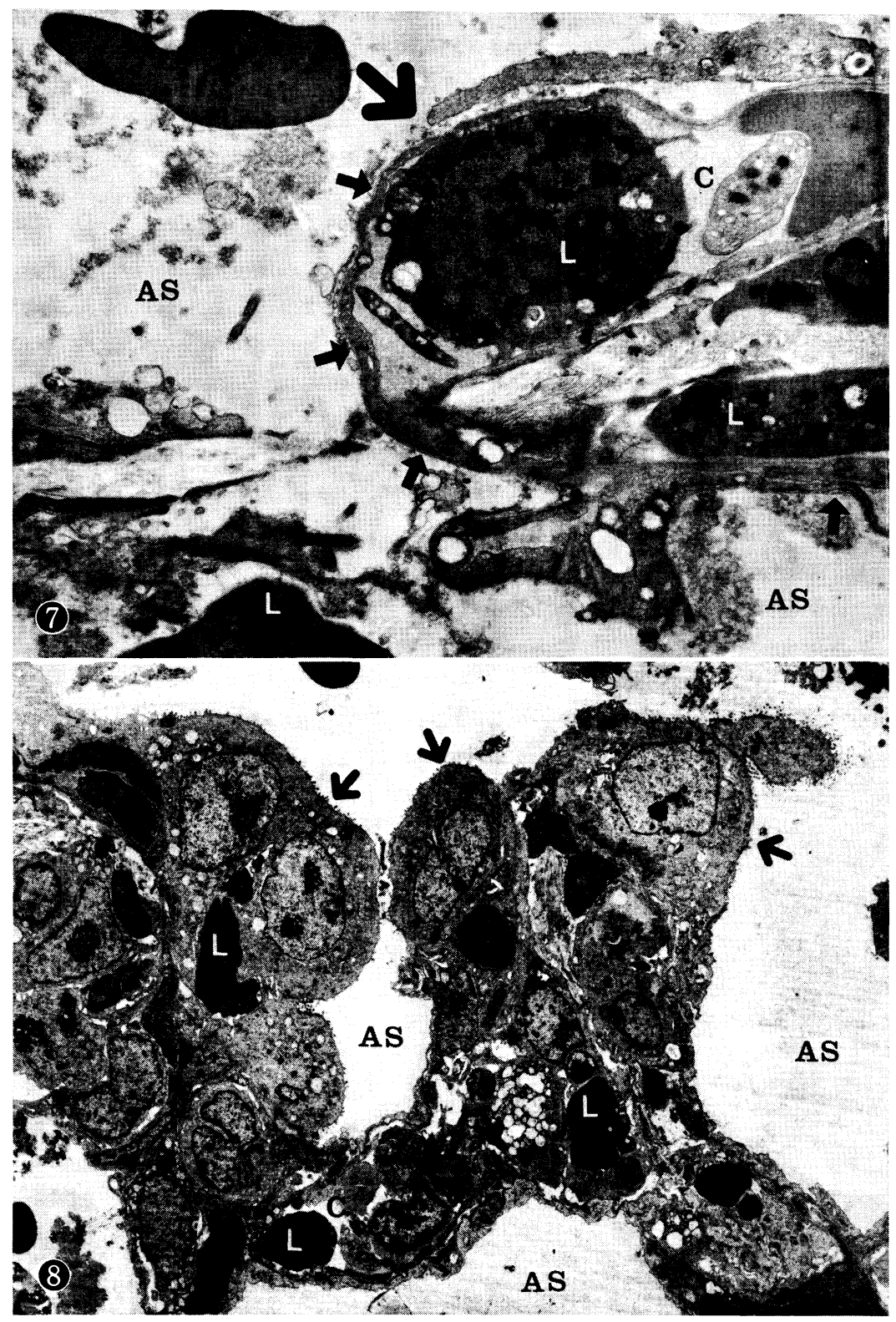

Fig. 7. An electron micrograph from the lung tissue at $5 \mathrm{hr}$ after $0.5 \%$ carrageenan treatment showing type 1 pneumocyte damage. Presence of damaged cell debris and floating of membranous cell process (large arrow) of type 1 pneumocyte in alveolar spaces (AS) is shown. The basement membrane is denuded (small arrow). C, capillary; L, leukocyte. $\times 10,000$.

Fig. 8. An electron micrograph from the lung tissue at $40 \mathrm{hr}$ after $0.5 \%$ carrageenan treatment showing proliferating immature type 2 pneumocytes (arrow) containing microvilli, conspicuous ribosomes and a few osmiophilic inclusions. $\times 3,600$. 


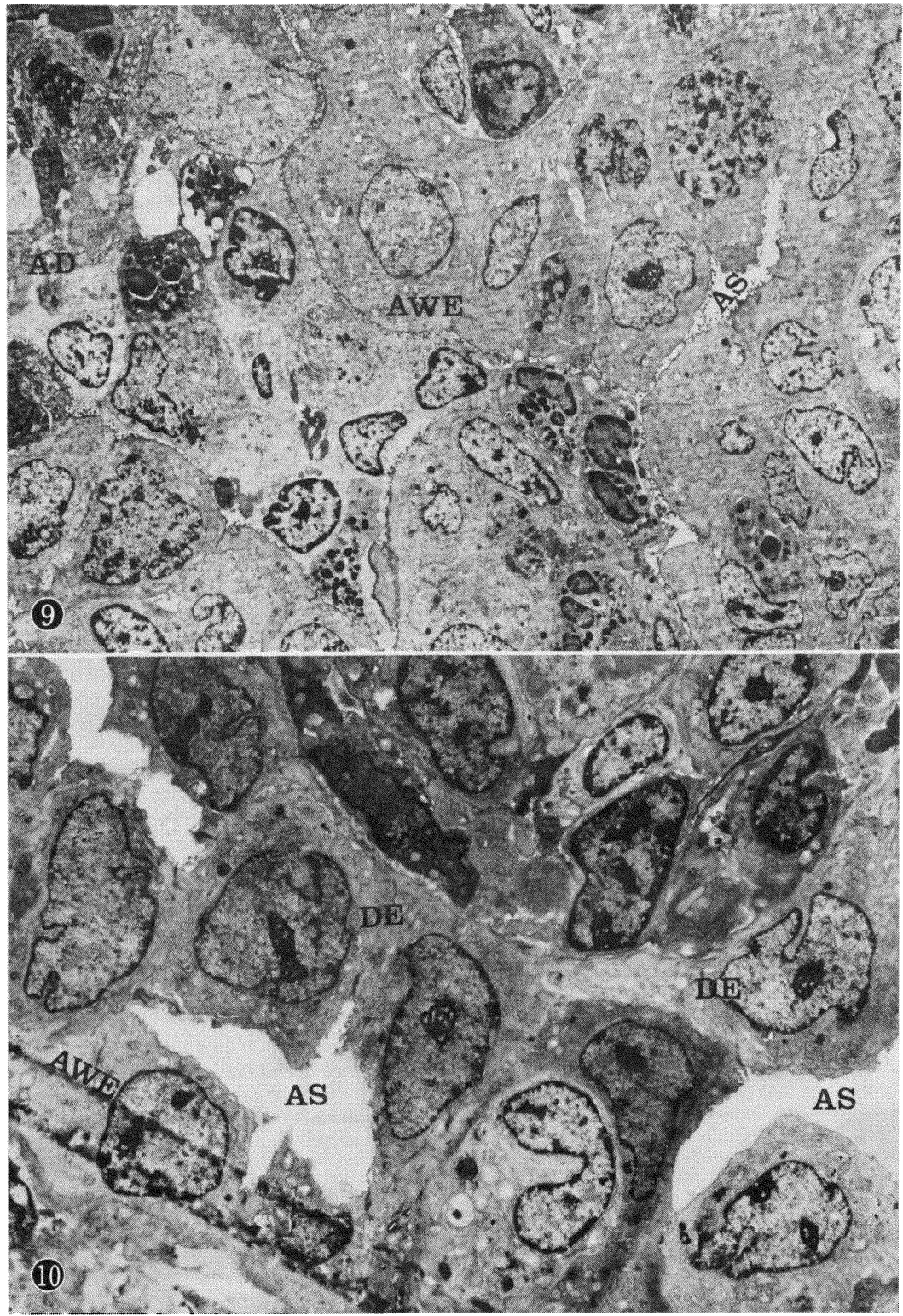

Fig. 9. An electron micrograph from the lung tissue at $60 \mathrm{hr}$ after $0.5 \%$ carrageenan treatment showing well marked proliferation of immature type 2 pneumocytes. AWE, alveolar wall lining epithelium;AS, alveolar space; $A D$, alveolar duct. $\times 3,600$.

Fig. 10. An electron micrograph of the marked rectangular area in Fig. 5 showing the continuity between alveolar wall lining epithelium (AWE) and desquamating epithelial cell sheet (DE) which packs up macrophages, degenerated cells and cell debris in alveolar lumen (AS). $\times 4,000$. 


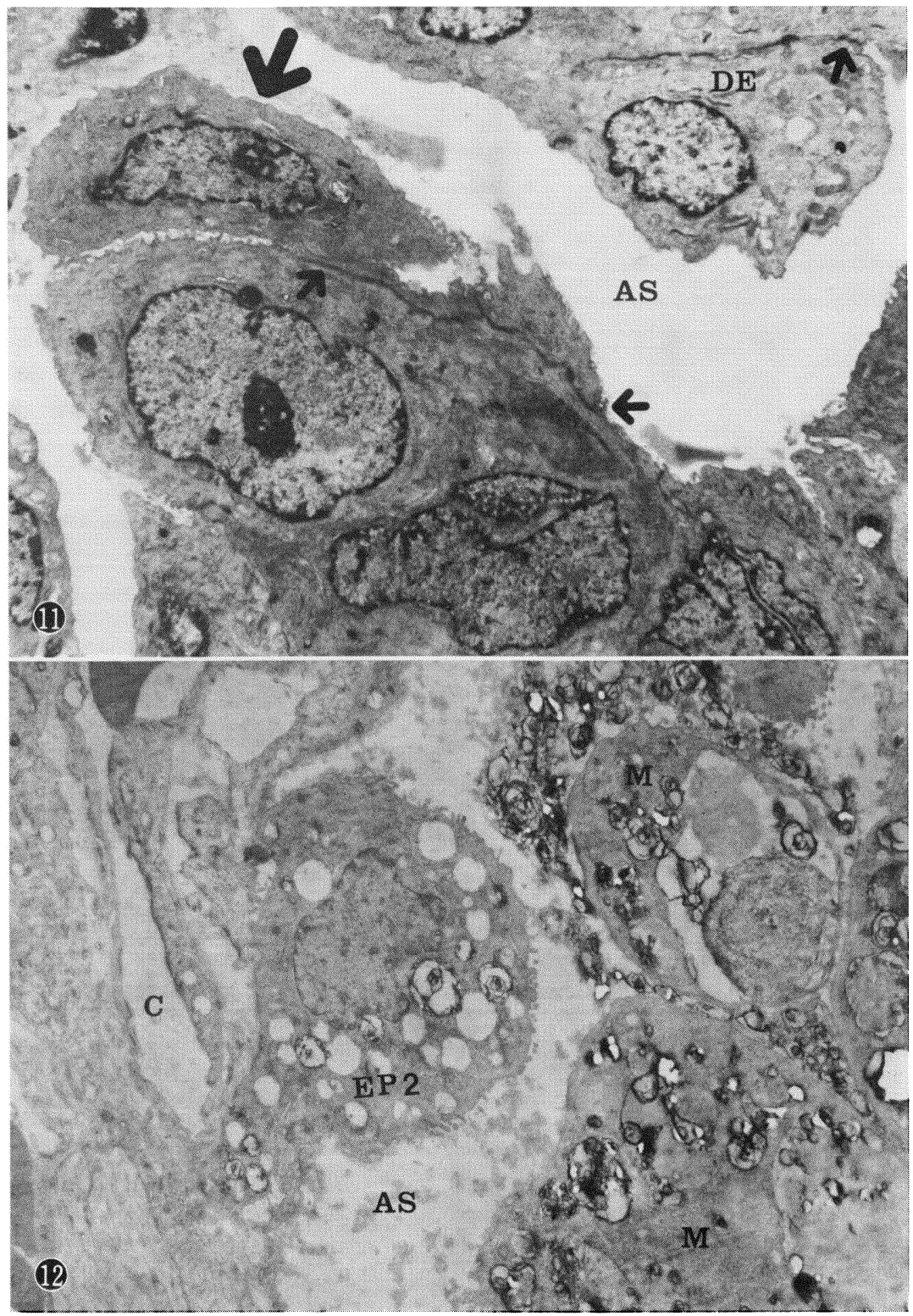

Fig. 11. An electron micrograph from the lung tissue on 5 days after $0.5 \%$ carrageenan treatment showing part of the alveolar wall lined by the proliferated immature type 2 pneumocytes. One (large arrow) of these pneumocytes seems to be free except for tight junctions (small arrow) with adjacent cells. Two degenerated epithelial cells located on the right upper corner are dislodged to be free in the alveolar space (AS). DE, desquamated epithelial cells. $\times 4,000$.

Fig. 12. An electron micrograph from the lung tissue at 3 weeks after $0.5 \%$ carrageenan treatment showing type 2 epithelial cell containing a few osmiophilic lamellar bodies, which is vacuolated, and degenerated alveolar macrophages $(\mathbf{M})$ with large vacuoles and osmiophilic fragments lying in contact with amorphous material containing numerous fine osmiophilic lamellar bodies. EP2, type 2 epithelial cell; C, capillary; AS alveolar space. $\times 6,300$. 
pack up the intra-alveolar cell debris and the many infiltrated alveolar macrophages as a cell mass, were also sloughing off (Fig. 10).

Observation at 2 and 3 weeks. Many alveoli were still filled predominantly with a large number of alveolar macrophages which had carrageenan containing vacuoles and osmiophilic materials in their cytoplasm. Sections from the animals which were sacrificed at 3 weeks showed that some alveoli were filled with proteinrich fluid containing fine osmiophilic materials admixing with the foamy macrophages. The alveolar lining epithelial cells were cuboidal or elliptical in shape in many alveoli and membranous in others. There was an infiltration of the interstitial spaces with mononuclear leukocytes and fibroblast-like mesenchymal cells (Fig. 6).

Electron microscopic examination of alveolar contents showed that it consisted of alveolar macrophages engulfing many lamellar osmiophilic lipoidal bodies, having a concentric arrangement, and foamy regions in their cytoplasm. Lamellar osmiophilic materials were also lying free amid amorphous debris in alveolar spaces (Fig. 12).

\section{Discussion}

Our preliminary study showed that various concentration of lambda carrageenan solution have similar but graduated effects on the lung parenchyma. Treatment with $0.5 \%$ carrageenan solution among them made the best effect of marked proliferation of alveolar lining epithelial cells in addition to an infiltration of inflammatory cells. When the animals were treated with $0.5 \%$ lambda carrageenan solution, the initial impact of the drug on the alveoli was reflected in the early occurrence of multifocal necrosis of type 1 pneumocyte, exudation of plasma fluid into the alveoli and inflammatory cell infiltration at $5 \mathrm{hr}$. This susceptibility of type 1 pneumocyte to injury is similar to that seen in toxic lung injury after oxygen (Adamson and Bowden 1974a), radiation (Adamson et al. 1970), bleomycin (Adamson and Bowden 1974b), lysolecithin (Aronson and Johns 1977) and butylated hydroxytoluene (Adamson et al. 1977). In our experiments the proliferative response of damaged lung tissue after administration of carrageenan was shown to be apparent by $40 \mathrm{hr}$ and to reach a maximum in 60 and $72 \mathrm{hr}$ followed by a decline as the tissue becomes repaired. Since it is now established that type 2 pneumocyte functions as a stem cell of alveolar lining epithelium and proliferation of this epithelial cell is now recognized to be a common, nonspecific adaptive reparative tissue reaction to many forms of toxic lung injury (Witschi 1976), type 2 pneumocyte proliferation in the lungs after administration of carrageenan in our work may be a reparative response to lung tissue injury. Similar recovery pattern of type 2 pneumocyte proliferation have been determined in the lungs of animals exposed to $\mathrm{O}_{2}$ (Kapanci et al. 1969; Adamson and Bowden 1974a), $\mathrm{NO}_{2}$ (Evans et al. 1973) and tobacco smoke (Wells and Lamerton 1975) and treated by intratracheal instillation of trypsin (Aronson et al. 1976), $\mathrm{FeCl}_{3}$ (Penha and Werthamer 1973) and $\mathrm{CdCl}_{3}$ (Stelzner et al. 1975). However, it 
should be emphasized here that the magnitude of type 2 cell proliferative response after carrageenan treatment appeared to be quantitatively prominent. The mechanism of such an intense cell proliferation in carrageenan lungs is unknown. Ortega et al. (1970) reported that chronic pulmonary edema was accompanied by proliferation of type 2 pneumocytes. In many instances of the above mentioned toxic lung damage followed by proliferation of type 2 pneumocytes, capillary engorgement, edema and congestion have been reported. It may be speculated that by contraries the edema and capillary engorgement induced by the lung damage may be one of the necessities of alveolar cell proliferation. Carrageenan has been a useful agent in inducement of inflammatory process and subsequent edema and capillary engorgement. It must be also considered that the carrageenan pneumonia showed obvious selective retention of long-lived macrophages in particular which contained carrageenan in their cytoplasm (Velo and Spector 1973). On the other hand, Watt and Marcus (1970) reported that administration of carrageenan orally induced well marked hyperplastic mucosal changes in the colon of rabbits. They stated that in their pathogenesis, although inflammation is undoubtedly an important factor and some of the hyperplastic lesions can perhaps be dismissed as a manifestation of reactive hyperplasia, the other lesions may have arisen per se in absence of any associated inflammatory process. In our work it may be considered that some of the hyperplastic changes represented the mitogenic effect of carrageenan on alveolar lining epithelium.

Another main point to emerge concerns the exfoliated alveolar lining cells that were recovered in the alveolar spaces together with an infiltrate of alveolar macrophages. The presence of cell masses in dilated alveolar duct (Fig. 4) suggested that their disappearance from alveoli was associated with elimination through the bronchial tree. It is well known that newly divided type 2 pneumocytes can serve as precursors of type 1 pneumocytes (Evans et al. 1973; Adamson and Bowden 1974a). However, Slauson et al. (1976) reported in lesions of type 2 cell proliferation in subacute changes of irradiation lung injury, similar cells were often noted in the alveolar spaces, suggesting that the proliferating cell occasionally sloughed off. Since much more desquamated epithelial cells in the alveolar spaces of the carrageenan lungs than those in irradiation lungs were demonstrated, the histological findings observed in those lungs appeared to be similar to those in human desquamative interstitial pneumonitis. However, desquamative interstitial pneumonitis is a disease of chronic interstitial pneumonia, but not an acute pneumonia.

A long term histological study on the carrageenan-induced lung inflammation, using high concentration ( $2 \%$ solution) of carrageenan, was performed by Bowers et al. (1980). They described that carrageenan-containing macrophages, but no other cell types, were observed in alveolar lumina at day 500 of their experiments. In our work, using $0.5 \%$ lambda carrageenan solution, observation at 3 weeks showed some alveoli be packed with proteinous fluid containing osmiophilic materials admixing with the foamy macrophages. Electron microscopic examination of 
alveolar contents showed that they were lamellar osmiophilic materials lying free amid amorphous debris and alveolar macrophages engulfing them. The condition resembled that seen in human alveolar proteinosis and could be considered to be comparable to drug-induced alveolar proteinosis (Witschi 1976). Heppleston and Young (1972) stated that both human and drug-induced alveolar proteinosis results from an initial type 2 pneumocyte hyperplasia which causes excessive production of phospholipid material combined with protein, and that this overwhelms the phagocytotic potential of the alveolar macrophages. Their interpretation is favorable of the light and electron microscopic findings observed in our experiments. We have not conducted detailed studies of long term effects of carrageenan on the lung tissue of surviving animals. However, on 150 days after carrageenan treatment there was some evidence of emphysematous lesions and a few alveoli were still filled up with macrophages.

\section{References}

1) Adamson, I.Y.R. \& Bowden, D.H. (1974a) The type 2 cell as progenitor of alveolar epithelial regeneration: a cytodynamic study in mice after exposure to oxygen. Lab. Invest., 30, 35-42.

2) Adamson, I.Y.R. \& Bowden, D.H. (1974b) The pathogenesis of bleomycin-induced pulmonary fibrosis in mice. Amer. J. Path., 77, 185-198.

3) Adamson, I.Y.R., Bowden, D.H. \& Wyatt, J.P. (1970) A pathway to pulmonary fibrosis: an ultrastructural study of mouse and rat following radiation to the whole body and hemithorax. Amer. J. Path., 58, 481-498.

4) Adamson, I.Y.R., Bowden, D.H., Cote, M.G. \& Witschi, H. (1977) Lung injury induced by butylated hydroxytoluene: cytodynamic and biochemical studies in mice. Lab. Invest., 36, 26-32.

5) Alley, A.R. \& Manktelow, B.E. (1971) Alveolar epithelization in ovine pneumonia. J. Path., 103, 219-224.

6) Aronson, J.F. \& Johns, L.W. (1977) Injury of lung alveolar cells by lysolecithin. Exp. mol. Path., 27, 35-43.

7) Aronson, J.F., Johns, L.W. \& Pietra, G.G. (1976) Initiation of lung cell proliferation by trypsin. Lab. Invest., 34, 529-536.

8) Bowers, R.R., Houston, F., Clinton, R., Lewis, M. \& Ballard, R. (1980) A histological study of the carrageenan-induced granuloma in the rat lung. J. Path., 132, 243-253.

9) Evans, M.J., Cabral, L.J., Stevens, R.J. \& Freeman, G. (1973) Renewal of alveolar epithelium in the rat following exposure to $\mathrm{NO}_{2}$. Amer. J. Path., 70, 175-190.

10) Heppleston, A.G. \& Young, A.E. (1972) Alveolar lipoproteinosis: an ultrastructural comparison of the experimental and human forms. J. Path., 107, 107-117.

11) Kapanci, Y., Weibel, E.R., Kaplan, H.P. \& Robinson, F.R. (1969) Pathogenesis and reversibility of the pulmonary lesions of oxygen toxicity in monkeys: II Ultrastructural and morphometric studies. Lab. Invest., 20, 101-118.

12) Leroy, E.P. (1969) The blood-air barrier in desquamative interstitial pneumonia (D.I. P.). Virchow Arch. Abt. A Path. Anat., 348, 117-130.

13) Merick, B. \& Reid, L. (1970) The alveolar wall. Brit. J. Dis. Chest, 64, 121-140.

14) Ortega, P., Uhley, H.N., Leeds, S.E., Friedman, M. \& Sampson, J.J. (1970) Serial electron and microscopic studies on the dog lung in chronic experimental pulmonary edema. Amer. J. Path., 60, 57-74.

15) Penha, P.D. \& Werthamer, S. (1973) The role of pneumocyte II in alveolar injury and repair. Amer. Rev. resp. Dis., 107, 1109.

16) Reynolds, E.S. (1963) The use of lead citrate at high $\mathrm{pH}$ as an electron-opaque 
stain in electron microscopy. J. Cell Biol., 17, 208-212.

17) Slauson, D.O., Hahn, F.F., Benjamin, S.A., Chiffelle, T.L. \& Jones, R.K. (1976) Inflammatory sequences in acute pulmonary radiation injury. Amer. J. Path., 82, $549-572$.

18) Stelzner, M.F., Baron, D.A. \& Esterly, J.R. (1975) Cadmium-induced lung injury. Lab. Invest., 35, 457.

19) Szereda-Przestaszewska, M. \& Sieiskiń, M. (1979) Lung pathology in carrageenan pneumonia of intact and chronically vagotomized rabbits. J. comp. Path., 89, 449456.

20) Trenchard, D., Gardner, D. \& Guz, A. (1972) Role of pulmonary vagal afferent nerve fibers in the development of rapid shallow breathing in lung inflammation. Clin. Sci., 42, 251-263.

21) Velo, G.P. \& Spector, W.G. (1973) The origin and turnover of alveolar macrophages in experimental pneumonia. J. Path., 109, 7-19.

22) Wachtlová, M., Chválová, M., Holuśa, R. \& Palećek, F. (1975) Carrageenaninduced experimental pneumonia in rats. Physiol. Bohemoslov., 24, 263-268.

23) Watt, J. \& Marcus, R. (1970) Hyperplastic mucosal changes in rabbit colon produced by degraded carrageenan. Gastroenterology, 59, 760-768.

24) Wells, A.B. \& Lamerton, L.F. (1975) Regenerative response of the rat tracheal epithelium after acute exposure to tobacco smoke: a quantative study. $J$. nat. Cancer Inst., 55, 887-889.

25) Witschi, H.P. (1976) Proliferation of type II alveolar cells: a review of common response in toxic lung injury. Toxicology, 5, 267-277. 